\title{
Controller structure for plants with combined saturation and stiction
}

DOI:

$10.1177 / 0959651818806410$

\section{Document Version}

Accepted author manuscript

Link to publication record in Manchester Research Explorer

\section{Citation for published version (APA):}

Rodriguez-Linan, M. D. C., \& Heath, W. (2018). Controller structure for plants with combined saturation and stiction. Institution of Mechanical Engineers. Proceedings. Part I: Journal of Systems and Control Engineering . https://doi.org/10.1177/0959651818806410

\section{Published in:}

Institution of Mechanical Engineers. Proceedings. Part I: Journal of Systems and Control Engineering

\section{Citing this paper}

Please note that where the full-text provided on Manchester Research Explorer is the Author Accepted Manuscript or Proof version this may differ from the final Published version. If citing, it is advised that you check and use the publisher's definitive version.

\section{General rights}

Copyright and moral rights for the publications made accessible in the Research Explorer are retained by the authors and/or other copyright owners and it is a condition of accessing publications that users recognise and abide by the legal requirements associated with these rights.

\section{Takedown policy}

If you believe that this document breaches copyright please refer to the University of Manchester's Takedown Procedures [http://man.ac.uk/04Y6Bo] or contact uml.scholarlycommunications@manchester.ac.uk providing relevant details, so we can investigate your claim.

\section{OPEN ACCESS}




\section{Controller structure for plants with combined saturation and stiction}

\author{
Ma. del Carmen Rodríguez-Liñán ${ }^{1}$ and William P. Heath²
}

\begin{abstract}
A compensation strategy for plants that suffer from both stiction and saturation in the input is proposed. The strategy exploits a principle established previously by the authors referred to as the 'saturation equivalence'. This approach shows that, for systems affected by a certain class of nonlinearities and saturation at the actuator, the addition of a right inverse of the nonlinearity, in series with the plant, results in a saturation. This paper shows that a stiction model belongs to this class of nonlinearities and that it can be compensated using the method of 'saturation equivalence' by implementing a right inverse for an approximate stiction model. Then, the resulting saturation can be treated by traditional methods, such as anti-windup or MPC. Results are demonstrated in a case study plant via simulation.
\end{abstract}

\title{
Keywords
}

Stiction compensation, saturation, anti-windup

\section{Introduction}

'Stiction' is a term formed from the combination of the words 'static' and 'friction'. It was coined to describe the effect that occurs when two surfaces in contact stick to each other, thus impeding movement. It refers to the threshold that is to be exceeded before the objects are released [1].

Stiction is common in control valves used in the process industry. When it is present, problems like increased energy consumption, decreased product quality, or excessive wear of the machinery may appear. It is mentioned in [2] that about $20-30 \%$ of the oscillations in control loops are caused by stiction. Hence, it is important to develop proper identification, analysis, and compensation schemes for this nonlinearity, [3, 4].

\section{Modelling and compensation strategies for stiction}

Following the literature, the stiction problem can be analyzed from two main fronts: identification and modeling, and compensation.

Models for stiction can be divided into two main types: first principle (or physical models) and data-driven models. Data-driven models are less descriptive than physical models and their parameters are empirically obtained. However, they are more commonly used because they do not need precise knowledge of the physical parameters; they use qualitative behavior of the valve instead. This way, data-driven models are more useful since their parameters are easy to define and to comprehend, [3, 5]. Numerous works exist in this respect, [3], [6-12], among others. The reader is also referred to [5] for a deep review on stiction modeling.

This work is concerned with stiction compensation; thus, this introduction will be focused on existing compensation methods rather than modeling.

Stiction compensation is a wide area of research in the literature. For instance Armstrong-Hèlouvry et al. [13] list about one hundred references in the topic up to 1994. Three main types of compensation strategies are mentioned: friction avoidance, non model-based compensation and model-based compensation. Typical applications, and the specific task each model focuses on, are also described. It should be noted, however, that [13] does not include a discussion on data-driven models; instead, only modelbased compensation techniques that use general friction models are considered. A review on stiction analysis, detection and compensation has been recently published by Bacci Di Capaci and Scali, [5]. In particular,

\footnotetext{
1 CONACyT-Instituto Tecnológico de Ensenada.

2 School of Electrical and Electronic Engineering, University of Manchester.

\section{Corresponding author:}

Ma. del Carmen Rodríguez-Liñán, Instituto Tecnológico de Ensenada, Boulevard Tecnológico 150, Ex-Ejido Chapultepec, 22780, Ensenada, Baja California, Mexico.

Email: macaroli270@yahoo.com.mx
} 
a comprehensive exploration of different compensation techniques is included, comparing results and assessing their efficiency.

Different compensation approaches based on either physical or data-driven models have been proposed. Since this work is based on the latter, the following discussion will be more heavily focused on controller design for datadriven models.

Non-model based stiction compensation strategies include 'dithering' and 'impulse control' [4], which are ineffective for pneumatic valves (the most common type found in control loops [7]) since they filter high frequency signals, [4, 7]. An alternative method is 'the knocker' introduced by Hägglund [4]. It consists of a series of short pulses added to the control signal until the valve is released. Over time, the valve will displace as the pressure on the actuator increases. The pulses are designed based on three configuration parameters chosen on a trial-and-error basis, [4].

Noting that the knocker has the disadavantage of adding stress to the valve stem, accelerating wear of the valve, Srinivasan and Rengaswamy [7] present the 'two-moves' approach, based on a one-parameter model. This method takes the stem to the steady-state value, and then uses a controller to prevent it from deviating from there. Several works have been based on this method, such as [6, 1417]. Ivan and Lakshminarayanan [6] extend the 'twomoves' approach using He's model [9] with an improved estimation scheme. The compensation is achieved via 'constant reinforcement', which is similar to the knocker, except that the signal added to the control law is constant. Farenzena and Trierweiler [14], based on the two-moves approach by [7], use a modified PI for processes with constant disturbances. Cuadros et al. [15] propose two methods that try to make up for the disadvantages of Srinivasan's approach; enhancements are made so that knowledge of the plant in not necessary, instead changes to set-point and disturbances are considered. In [16] an open-loop scheme is proposed to eliminate oscillations caused by stiction. This method does not require knowledge of the actual valve position. Later, Tang and Wang [17] present a solution for the estimation of the desired controller output for a given desired valve position, thus reducing the computation time associated with the two-moves method.

Mohammad and Huang [18] propose a method to eliminate or reduce oscillations due to stiction by controller tuning. In particular, they focus on P or PI controllers. An analysis of the limit cycles induced by stiction presented in [19] leads to a formulation that permits to analytically tune a PID controller to compensate stiction. This is achieved by relating the PID parameters to the oscillations caused by stiction, thereby limiting the nonlinearity's effect on the plant through the PID controller; results are demonstrated in simulation.

Model Predictive Controllers (MPC) have also been proposed for stiction compensation. Zabiri and Samyudia [20] proposed in 2009, the first of such approaches: an MPC controller based on a Mixed-Integer Quadratic Program (MIQP). In [21] the authors present an Economic Model Predictive Control (EMPC) approach to compensate valve stiction. The dynamics of the valve are modelled from the forces acting on it. The evidence demonstrating this method is shown by simulation examples. Bacci Di Capaci et al. [22] present three different MPC strategies for valve siction compensation based on He's model [9]. The studied methods include a linear MPC, a nonlinear MPC embedded with the sticking valve model, and a linear MPC combined with an inverse model of the stiction nonlinearity. The authors propose a smooth representation of the discontinuities present in stiction. Novak and Chalupa [23] present an MPC controller, also based on MIQP, to compensate for deadzone in a valve. The method is demonstrated in a two-tank level control system. Finally, Rodríguez and Heath [24] propose a simple MPC method, based on a Convex Quadratic Program, for the compensation of nonlinearities such as deadzone, backlash, and stiction, in combination with saturation. The approach is novel, since not much attention has been given in the literature to the effects of saturation in sticking actuators. The method proposes that, because of the 'saturation equivalence' principle introduced by the authors in 25 , 26], a simple MPC, with only saturation bounds, can compensate for the nonlinear effects. In [27], the 'saturation equivalence' approach was theoretically and experimentally demonstrated for backlash nonlinearity. The aim of this work is to show and formalize such principle for stiction nonlinearity.

\section{Stiction and saturation}

This paper describes a novel compensation method for a linear plant affected by saturation and stiction in series with the actuator. It is proposed that, if a right inverse of an approximate stiction can be found and placed in series with the plant's nonlinearities (namely, saturation and stiction), then the problem can be seen as an input saturated system. This property, called 'saturation equivalence' was introduced by the authors in [25], and formalized in [27] for backlash.

To the best of the authors' knowledge, there have not been reported in the literature dedicated control strategies for combined stiction and saturation at the actuator. The contributions of this work are thus listed below. 


\section{Contributions}

1. Stiction inverse. An approximation to stiction $\tilde{\varphi}_{c, J}$ is considered. The right inverse to this approximation $\tilde{\varphi}_{c, J}^{+}$is constructed. It is shown that

$$
\tilde{\varphi}_{c, J} \tilde{\varphi}_{c, J}^{+} y=y .
$$

This inverse is used as a compensation structure for stiction. Note that since in real life stiction will not present infinite jumps (as in the 'ideal' case), then it is possible to approximate stiction and to calculate a right inverse for it. Then, the inverse can be used to design a compensator.

Remark 1. The inverse $\tilde{\varphi}_{c, J}^{+}$is constructed using a parameter $\varepsilon$ to slant the jumps of the stiction. This is, $\tilde{\varphi}_{c, J} \rightarrow \varphi_{c, J}$ with $\varepsilon$ sufficiently small. Then, the parameter $\varepsilon$ is interpreted as a control parameter used to improve the compensation.

2. Equivalence to saturation. Following the results in [27], a class $\Phi_{c}$ of nonlinearities is defined such that if $\varphi_{c} \in \Phi_{c}$, then

$$
\varphi_{c} \sigma_{d} \varphi_{c}^{+}=\sigma_{d-c},
$$

where $\varphi_{c}^{+}$is the right inverse of $\varphi_{c}$ and $d>c>0$.

It is shown that the approximate stiction $\tilde{\varphi}_{c, J}$ belongs to $\Phi_{c}$.

Remark 2. The 'saturation equivalence' property holds exactly when $\tilde{\varphi}_{c, J}^{+}$is the exact inverse of $\tilde{\varphi}_{c, J}$. Note that it is possible to use $\tilde{\varphi}_{c, J}^{+}$since, in real life systems, stiction will not have infinite jumps, i.e. $\varphi_{c, J}$ will not happen in real life.

3. Optimality of structure It is also shown that the 'saturation equivalence' (2) is optimal.

Contributions 2 and 3 derive from previous results presented by the authors in [27] for backlash nonlinearity. In earlier works by the authors, the 'saturation equivalence' is stated [24-26] for deadzone, backlash and stiction, and later, formalized [27] for backlash. The purpose and main contribution of this work is to demonstrate and formalize the application of said principle for the stiction nonlinearity, especially considering that it constitutes a non invertible operator.

4. Application with anti-windup. If $\tilde{\varphi}_{c, J} \in \Phi_{c}$, then it is possible to construct control laws based on standard anti-windup for plants subject to a nonlinearity $\varphi_{c, J}$ and a saturation $\sigma_{d}$ in the actuator. This is demonstrated with a basic anti-windup structure combined with a nonlinearity $\tilde{\varphi}_{c, J}^{+}$.

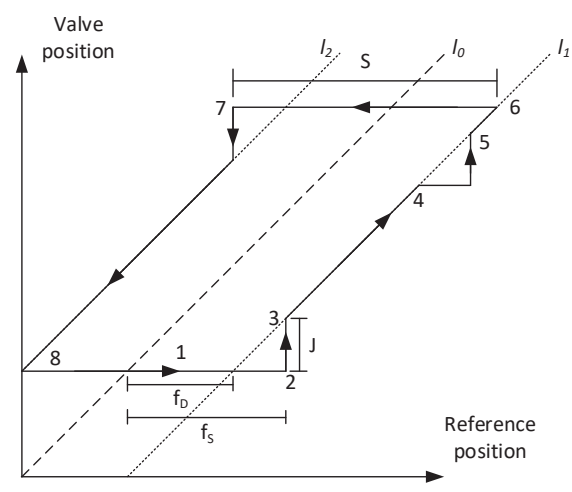

Figure 1. Valve Stiction. Points 1 to 8 represent different instants in the sticking valve's behaviour, while line $l_{0}$ identifies the ideal behaviour, when the valve does not stick.

\section{Stiction inversion}

Stiction plus saturation is common in practice but not much considered in the literature. This paper tackles this problem via inversion of the stiction characteristic, assuming a simple two-parameter model for it. Compensation of saturation and stiction in a plant is dealt with by adding a right inverse of the approximate stiction in series with the saturation and stiction nonlinearities. Such interconnection yields a saturation, as will be shown later.

\section{Stiction behaviour and model}

In this work, stiction is modelled using a representation based on the data-driven model presented in [8]. A compensation strategy that follows the inverse approach by [28] for deadzone and backlash is proposed. The motivation for this is that stiction behaviour resembles that of backlash, see Fig.1.

The behavior of a valve with stiction is presented in Fig. 1 It can be divided into three types of movement: a moving phase (represented by lines $l_{1}$ and $l_{2}$ ), a sticking phase (section $S=f_{D}+f_{S}$ ), and a slip-jump phase (indicated by $J)$.

Referring to Fig. 1] if the valve is initially at rest at point 1 , stiction will prevent it from moving linearly along the ideal line $l_{0}$. Once the valve input is strong enough to overcome stiction (point 2), the valve is abruptly opened, generating a jump $J=f_{S}-f_{D}$ (point 3 ). The valve, now released, follows the input along line $l_{1}$, unless it gets stuck again (points 4 and 5). Point 6 represents the instant when the input changes direction to close the valve. Because of stiction, the valve will not move for a lapse $S=f_{D}+f_{S}$. When the force is large enough, the valve will move (point 7) with a jump $J$. If there are no other obstacles along the way, the valve will continue moving along line $l_{2}$. 


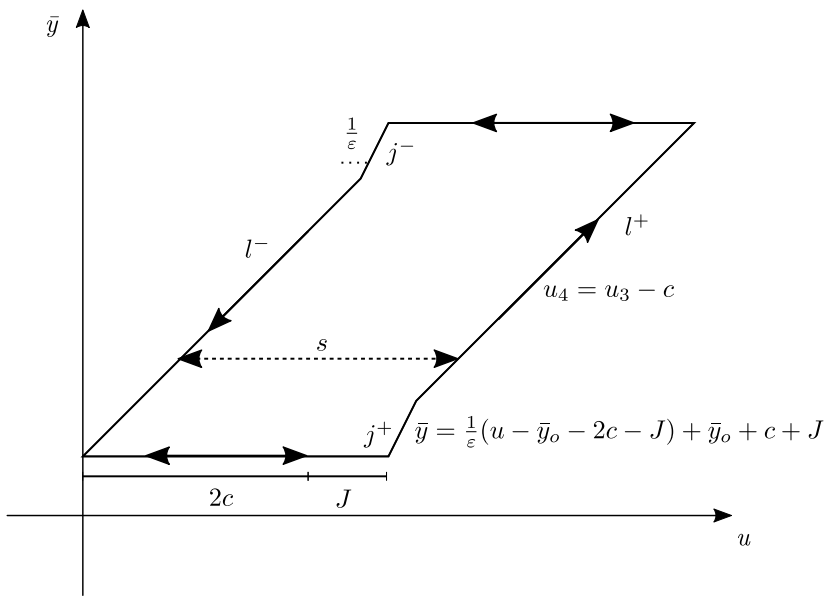

Figure 2. Approximate stiction. The segments $\left\{l^{+}, l^{-}, s, j^{+}, j^{-}\right\}$ indicate linear, slipping and jumping behaviour. Additionally, the values taken by $\bar{y}$ at each positive transition segment are indicated, the values corresponding to the rest of the transitions values can be deduced by symmetry.

In what follows $f_{D}$ will be referred to as $c$ to comply with the results reported in [27].

\section{Approximate stiction model}

Observing Fig. 1 we note that the stiction characteristic features discontinuous jumps, which we denote by $J$. Then, the stiction nonlinearity $\varphi_{c, J}: u \rightarrow \bar{y}$ does not have a right inverse. Therefore, an approximate stiction, that smooths the jumps $J$, and its inverse are defined. To do this, a new parameter $\varepsilon$ is used to characterise the approximations. The approximate stiction model shown in Fig. 2 tends to that in [8] when $\varepsilon$ is sufficiently small. Fig 2] shows the behaviour of the approximate stiction. In the figure, the labels $\left\{l^{+}, l^{-}, s, j^{+}, j^{-}\right\}$are used to indicate linear, slipping and jumping behaviour.

Next, a model for the approximate stiction is defined. Let $\tilde{\varphi}_{c, J}$ be the nonlinear operator representing the approximate stiction nonlinearity. The approximate stiction is parameterised with respect to the exact sticion by the design parameter $\varepsilon$, which defines the slope of the jump $J$. Thus, the model is defined using parameters $c, J$ and $\varepsilon$, see Fig. 2,

Then, $\tilde{\varphi}_{c, J}$ is an operator that represents the approximate stiction nonlinearity with parameters $J, c, \varepsilon>0$ and is described as follows:

$$
\dot{\bar{y}}(t)= \begin{cases}\dot{u}(t) \quad & \text { if } \dot{u}(t)>0 \text { and } u(t) \geq \bar{y}(t)+c, \\ & \text { if } \dot{u}(t)<0 \text { and } u(t) \leq \bar{y}(t)-c, \\ 0 & \text { if } \dot{u}(t)=0 \text { and } \bar{y}(t)-c<u(t)<\bar{y}(t)+c, \\ \frac{1}{\varepsilon} \dot{u}(t) & \text { if } \dot{u}(t)>0 \text { and } u(t) \leq \bar{y}(t)-c \\ & \text { and } u(t)<\bar{y}_{o}+2 c+J, \\ & \text { if } \dot{u}(t)<0 \text { and } u(t) \geq \bar{y}(t)+c \\ & \text { and } u(t)>\bar{y}_{o}-2 c-J,\end{cases}
$$

where $\bar{y}_{o}$ refers to the value of output $y(t)$ at the instant when $u(t)$ changes direction.

In the next section a right inverse for the approximate stiction model is presented.

\section{Inverse of the approximate stiction model}

Consider the operator $\tilde{\varphi}_{c, J}^{+}: y \rightarrow u$ and let it represent the right inverse of the approximate stiction model presented in the previous section. The behaviour of the inverse stiction is shown in Fig. 3. The values taken by $u(t)$ at each segment of the nonlinearity are illustrated in the same figure.

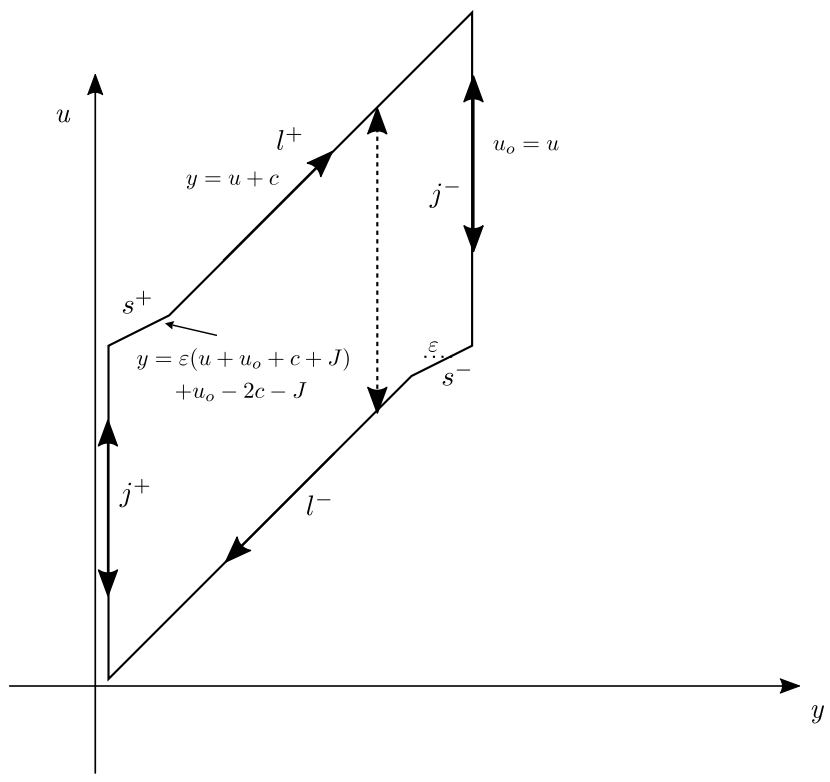

Figure 3. Approximate inverse stiction. The segments $\left\{l^{+}, s^{+}, j^{+}, l^{-}, s^{-}, j^{-}\right\}$indicate linear, slipping and jumping behaviour. The values taken by $y(t)$ at each positive transition segment are indicated accordingly, while the rest of the transitions values can be obtained by symmetry.

Then, $\tilde{\varphi}_{c, J}^{+}$is an operator that represents the right inverse of the approximate stiction nonlinearity with parameters $J, c, \varepsilon>0$ as follows: 


$$
\dot{u}(t)= \begin{cases}\dot{y}(t) & \text { if } \dot{y}(t)>0 \text { and } y(t) \geq u(t)-c, \\ & \text { if } \dot{y}(t)<0 \text { and } y(t) \leq u(t)+c, \\ \varepsilon \dot{y}(t) & \text { if } \dot{y}(t)=0, \\ & \text { if } \dot{y}(t)<0 \\ & \text { and } y_{o}-J-c \leq y(t)<u(t)-c, \\ & \text { if } \dot{y}(t)>0 \\ & \text { and } u(t)+c<y(t) \leq y_{o}+c+J, \\ g(t, t) & \text { if } \dot{y}(t)>0 \text { and } y(t)>u(t)+c, \\ -g(t, t) & \text { if } \dot{y}(t)<0 \text { and } y(t)<u(t)-c .\end{cases}
$$

with $g(\tau, t)=\delta(\tau-t)(2 c+J)$, where $\delta(t)$ as the Dirac- $\delta$ function. Thus $g(t, t)$ in equation (4) indicates that $g(\tau, t)$ is illustrated with $\tau=t$. Additionally, note that notation $y_{o}$ indicates the value of input $y$ before it changes direction.

\section{Invertibility of the approximate stiction characteristic}

Next, it is shown that the proposed inverse described above is the right inverse of the approximate stiction operator defined previously. This is $\tilde{\varphi}_{c, J} \tilde{\varphi}_{c, J}^{+} y=y$ In what follows, assume that $u=\tilde{\varphi}_{c, J}^{+}(y)$, and that $\bar{y}=\tilde{\varphi}_{c, J}(u)$.

Lemma 1. The operator $\tilde{\varphi}_{c, J}^{+}$is the right inverse of the approximate stiction $\tilde{\varphi}_{c, J}$, in the sense that

$$
\left(\tilde{\varphi}_{c, J} \tilde{\varphi}_{c, J}^{+}\right) y=y .
$$

Proof. First, suppose that $\dot{y}(t)>0$ for $t \in\left[t_{0}, t_{1}\right]$ and $t_{1}>t_{0}$. If $y\left(t_{0}\right) \geq u\left(t_{0}\right)-c$ and $u\left(t_{0}\right) \geq \bar{y}\left(t_{0}\right)+c$, then from (3) and (4), it follows that $\dot{\bar{y}}(t)=\dot{u}(t)=\dot{y}(t)$ for $t \in\left[t_{0}, t_{1}\right]$. Moreover $\bar{y}(t)=u(t)-c$ and $u(t)=y(t)+c$. Therefore, $\overline{y_{0}}(t)=u\left(t_{0}\right)-c=$ $y\left(t_{0}\right)+c-c=y\left(t_{0}\right)$. Hence, $\tilde{\varphi}_{c, J} \tilde{\varphi}_{c, J}^{+} y=y$ for any $t \in\left[t_{0}, t_{1}\right]$. On the other hand, if $y\left(t_{0}\right)=u\left(t_{0}\right)-c$ and $\bar{y}\left(t_{0}\right)-c<u\left(t_{0}\right)<$ $\bar{y}\left(t_{0}\right)+c$, then, according to (4), $u(t)$ will have a jump at $t=t_{0}$ so that $u(t)=\varepsilon\left(y-y_{o}+c+J\right)+y_{o}-2 c-J$ for $t=t_{o}^{+}$, where $y_{o}=y\left(t_{0}\right)$ is the recorded value of $y(t)$, at the time of the jump. Similarly, we define $\bar{y}_{o}\left(t_{0}^{+}\right)=\bar{y}\left(t_{0}\right)=y\left(t_{0}\right)$, from the previous analysis. The jump in $u(t)$ forces $\bar{y}(t)$ to traverse an inner segment so that $\bar{y}\left(t_{0}^{+}\right)=\frac{1}{\varepsilon}\left(u\left(t_{0}^{+}\right)-\bar{y}_{o}+2 c+J\right)+\bar{y}_{o}-c-$ $J$. Then $\bar{y}\left(t_{0}^{+}\right)=y\left(t_{0}^{+}\right)$, and $\tilde{\varphi}_{c, J} \tilde{\varphi}_{c, J}^{+} y=y$ for any $t \in\left[t_{0}, t_{1}\right]$.

When $\dot{y}(t)<0$ a similar analysis shows that $\tilde{\varphi}_{c, J} \tilde{\varphi}_{c, J}^{+} y=y$ for any $t \in\left[t_{0}, t_{1}\right]$.

If $\dot{y}(t)=0$ for $t \in\left[t_{0}, t_{1}\right]$, then $\tilde{\varphi}_{c, J} \tilde{\varphi}_{c, J}^{+} y=y$ holds for any $t \in\left[t_{0}, t_{1}\right]$.

If $\dot{y}(t)$ changes sign at $t=t_{1}$ then we can repeat the procedure and show that $\tilde{\varphi}_{c, J} \tilde{\varphi}_{c, J}^{+} y=y$ for any $t \geq t_{0}$.

\section{Example}

In this section, an example of stiction compensation is presented. It is based in an example taken from [10], where a level control system subject to stiction is considered; here it is used to illustrate how the proposed inverse stiction serves to compensate the stiction nonlinearity. The closed loop system is shown in Fig. 4

The flow $P_{F}(s)$ and the level dynamics $P_{L}(s)$ are given as

$$
P_{F}(s)=\frac{1}{0.2 s+1} \quad P_{L}(s)=\frac{1}{15 s} e^{-s} .
$$

Three cases are assumed:

Case 1 No stiction.

Case 2 Mild stiction in the input

(a) with PI control,

(b) with the proposed inverse stiction compensation.

Case 3 Strong sticion in the input

(a) with PI control,

(b) with the proposed inverse stiction compensation.

The corresponding parameters for each case are shown in Table 1.

Table 1. Stiction parameters

\begin{tabular}{ccc}
\hline Case & Kano et al. [10] & Stiction inverse \\
\hline 1 & $S=0, J=0$ & $c=0, J=0$ \\
2 & $S=1, J=0.3$ & $c=0.35, J=0.3$ \\
3 & $S=5, J=1$ & $c=2, J=1$ \\
\hline
\end{tabular}

In Case 1 it is assumed that no stiction is affecting the actuator. This is presented for comparison purposes, to show the natural behaviour of the plant, and the expected behaviour of the sticking plant when using a compensation method for stiction. A PI controller is used as a control unit, with parameters $K_{p}=1$ and $T_{i}=50$, where time is given in minutes [10].

For Cases 2 and 3, to compensate for stiction, a stiction inverse is added to the controller, such that the control unit in Fig. 4 is now given as shown in the block diagram of Fig. 5

Case 1: No stiction is present at the actuator. As mentioned before, this case is shown solely for comparison purposes, to illustrate the natural behaviour of the system when stiction is absent from the input. The closed-loop response and the control signal from the PI are shown in Fig. 6

Case 2(a): Mild stiction with PI. Here it is assumed that the linear plant (6) is affected by a mild stiction. To illustrate the benefits of the proposed inverse stiction compensation, the plant is first simulated in closed-loop with a standard PI controller, and no compensation for the nonlinearity. It can be observed in Fig.7 that oscillations are present around the reference in steady state, due to stiction. 


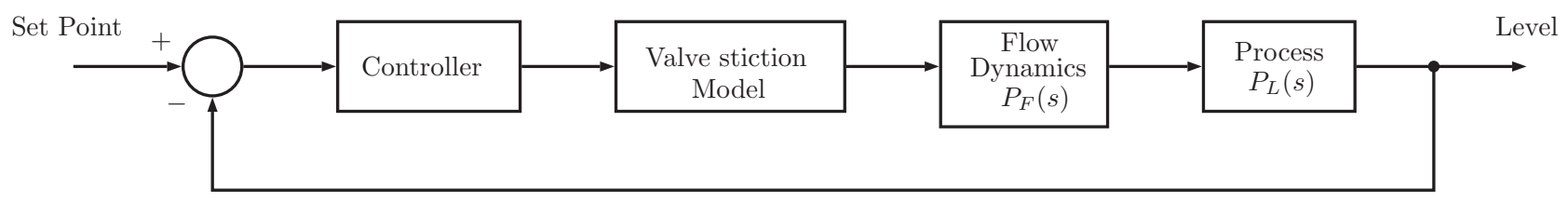

Figure 4. Block diagram of a level control system.

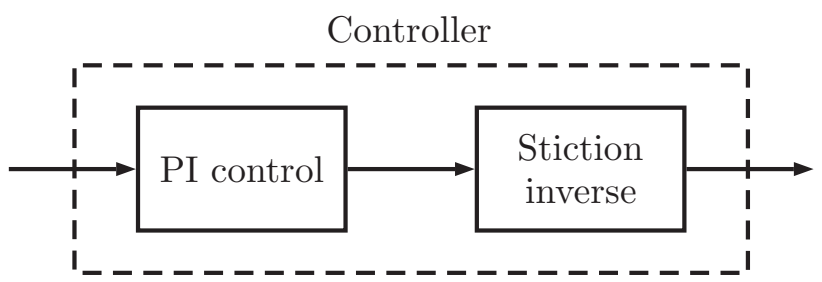

Figure 5. Controller unit for Cases 2 and 3. It comprises a PI controller and an inverse stiction compensator.
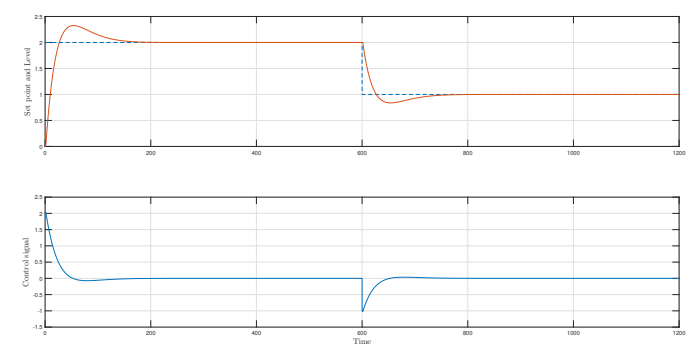

Figure 6. Case 1. Closed-loop response for level control system using a PI controller when no stiction is present at the actuator. The top plot shows the plant's output and the bottom figure shows the control signal.

Case 2(b): Mild stiction with proposed inverse stiction compensation. In this case, the plant is assumed to be affected by a mild stiction, but the proposed inverse stiction is used to compensate for the nonlinearity. The results can
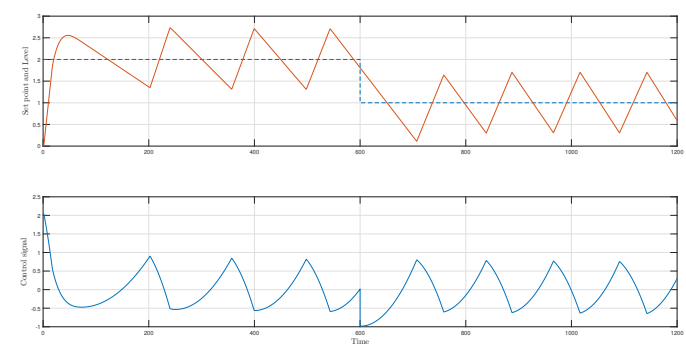

Figure 7. Case 2(a). Closed-loop response (top) for level control system using a standard PI controller and no stiction compensation, when mild stiction is affecting the actuator, and corresponding control signal. (bottom) be observed in Fig. 8, It can be observed that the output level correctly follows the reference. The approximate inverse stiction is described by parameter $\varepsilon=1 \times 10^{-4}$.
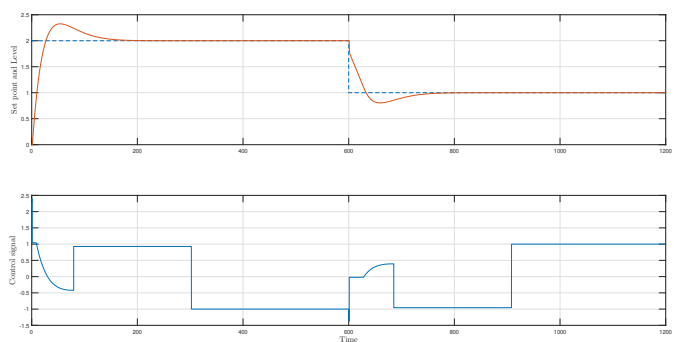

Figure 8. Case 2(b). Closed-loop response (top) for level control system using the proposed inverse stiction compensation, when mild stiction is affecting the actuator, and the corresponding control signal (bottom).

Case 3(a): Strong stiction with PI control. Now, it is assumed that the stiction nonlinearity is stronger, and that the plant is controlled by a standard PI. The corresponding closed-loop response and control signal are shown in Fig. 9 Note that the oscillations around the set point in this case are more severe.

Case 3(b): Strong stiction with proposed inverse stiction compensation. Now, the proposed inverse stiction is used to compensate for the stiction nonlinearity with larger parameters. As expected, the approximate inverse allows the output to follow the reference satisfactorily, despite the harshness of the stiction nonlinearity, see Fig. 10. As for Case 2(b), $\epsilon=1 \times 10^{-4}$. It should be noted that, provided the stiction nonlinearity is perfectly estimated, the stiction inversion is always effective. This is clear from Figs. 8 and 9 for mild and strong stiction; the response in both cases is essentially the same, regardless of the severity of the stiction nonlinearity.

\section{Saturation equivalence}

The results in this section derive from previously reported results from the authors in [27] for the backlash nonlinearity. In what follows, it is assumed that a linear plant $G$ is subject to saturation and stiction in the actuator, see Fig. 11, The authors have shown that the combination of the inverse nonlinearity with a saturation and the 

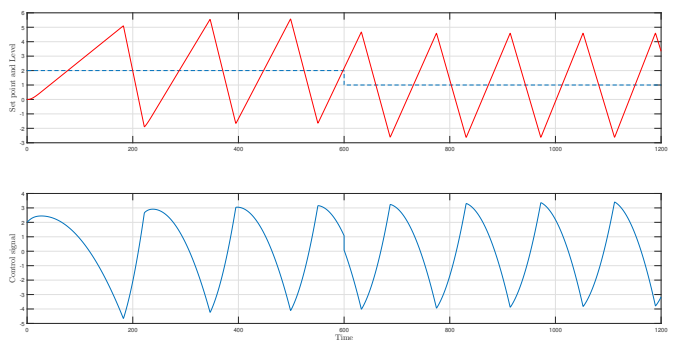

Figure 9. Case 3(a). Closed-loop response for level control system using a standard PI controller and no stiction compensation, when strong stiction is affecting the actuator (top), and corresponding control signal (bottom).
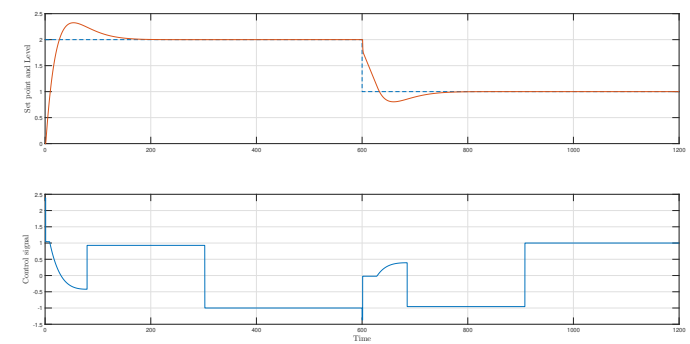

Figure 10. Case 3(b). Controller output (top) using a PI controller and a stiction inverse compensator, when a strong stiction is affecting the actuator. The bottom figure shows the control signal from the right inverse stiction.

nonlinearity itself results in a saturation of a different level. This result was referred to as the 'saturation equivalence' principle, [27].

The main result of this paper is to show that the 'saturation equivalence' result holds for the approximate stiction nonlinearity, as illustrated in Fig. 11

Saturation nonlinearity Let $\sigma_{d}$ be the operator mapping $v$ to $w$, characterized as

$$
w(t)=\operatorname{sat}_{d}(v(t))
$$

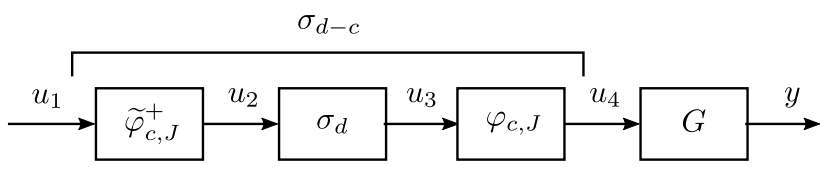

Figure 11. Saturation equivalence structure and plant dynamics. The block $\sigma_{d}$ represents actuator saturation and $\varphi_{c, J}$ represents the stiction nonlinearity. The block $G$ represents the remaining dynamics, assumed to be linear. The series interconnection of the right inverse of the approximate stiction nonlinearity $\tilde{\varphi}_{c, J}^{+}$, a saturation, and the nonlinear operator $\varphi_{c, J}$ is equivalent to a saturation $\sigma_{d-c}$.

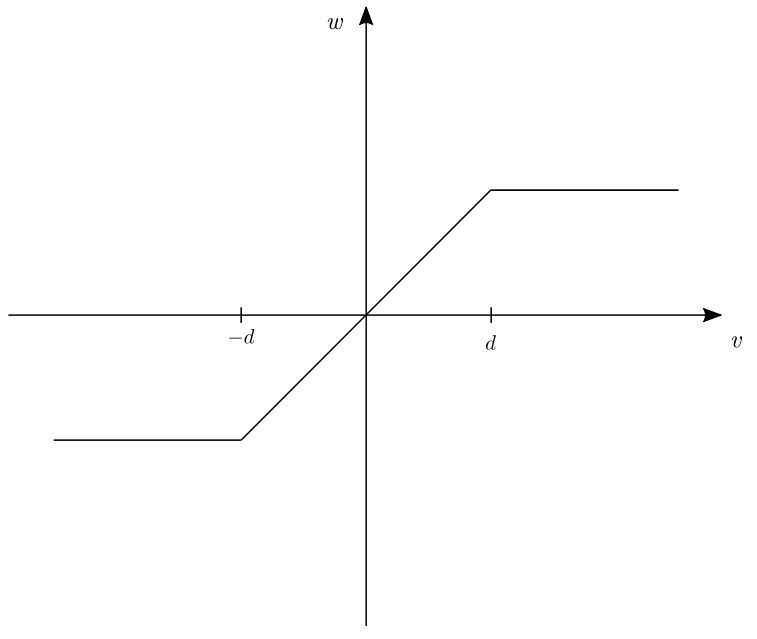

Figure 12. Saturation.

where

$$
\operatorname{sat}_{d}(v(t))=\left\{\begin{aligned}
d, & \text { if } v(t)>d \\
v(t), & \text { if }|v(t)| \leq d \\
-d, & \text { if } v(t)<-d
\end{aligned}\right.
$$

with $d>0$.

Theorem 1. Suppose for all continuous and differential signals $u$

$y=\tilde{\varphi}_{c, J}(w), w=\sigma_{d}(v)$ and $v=\tilde{\varphi}_{c, J}^{+}(u)$, with $d>c>0$.

Then if

$$
y\left(t_{0}\right)=\operatorname{sat}_{d-c}\left(u\left(t_{0}\right)\right) \text { for some } t_{0}
$$

then

$$
y(t)=\operatorname{sat}_{d-c}(u(t)) \text { for all } t \geq t_{0} .
$$

Proof. Three cases are considered.

Case 1. Suppose $|u(t)| \leq d-c$ for some interval $\left[t_{1}, t_{2}\right]$. Then, $w(t)=v(t)$ for all $t \in\left[t_{1}, t_{2}\right]$. It follows that if $y\left(t_{1}\right)=u\left(t_{1}\right)$, then $y(t)=u(t)$ for all $t \in\left[t_{1}, t_{2}\right]$.

Case 2. Suppose $u(t) \geq d-c$ for some interval $\left[t_{1}, t_{2}\right]$. Then $v(t) \geq d-2 c$ and $d-2 c \leq w(t) \leq d$ for all $t \in\left[t_{1}, t_{2}\right]$. If $y\left(t_{1}\right)=d-c$, then $\dot{y}(t)=0$ for all $t \in\left[t_{1}, t_{2}\right]$ and hence $y(t)=d-c$ for all $t \in\left[t_{1}, t_{2}\right]$.

Case 3. Suppose $u(t) \leq-(d-c)$ for some interval $\left[t_{1}, t_{2}\right]$. Then, similar to Case 2, $y(t)=-(d-c)$ for all $t \in$ $\left[t_{1}, t_{2}\right]$.

Remark Note that, the above holds when the inverse of the approximate stiction is at the segments $s^{+}$and $s^{-}$(and approximate stiction is at $j^{+}, j^{-}$, respectively).

To complete the proof the transitions between levels must be considered. Only transitions between when $|u(t)| \leq$ 
$d-c$ and $u \geq d-c$ (in both directions) will be considered. Conditions for transitions between when $|u(t)| \leq d-c$ and $u(t) \leq-(d-c)$ follow by symmetry. Since $u(t)$ is continuous these are sufficient to show that $(10) \Rightarrow 11$.

Transition 1. Suppose $|u(t)| \leq d-c$ for some interval $\left[t_{1}, t_{2}\right]$ and $u(t) \geq d-c$ for some interval $\left[t_{2}, t_{3}\right]$. If $y\left(t_{1}\right)=$ $u\left(t_{1}\right)$ then $y(t)=u(t)$ for all $t \in\left[t_{1}, t_{2}\right]$; in particular $y\left(t_{2}\right)=$ $d-c$. Hence $y(t)=d-c$ for all $t \in\left[t_{2}, t_{3}\right]$.

Transition 2. Suppose $u(t) \geq d-c$ for some interval $\left[t_{1}, t_{2}\right]$ and $|u(t)| \leq d-c$ for some interval $\left[t_{2}, t_{3}\right]$. If $y\left(t_{1}\right)=d-c$ then $y(t)=d-c$ for all $t \in\left[t_{1}, t_{2}\right]$; in particular $y\left(t_{2}\right)=u\left(t_{2}\right)$. Hence $y(t)=u(t)$ for all $t \in\left[t_{2}, t_{3}\right]$.

With some slight abuse of notation, it can be said that

$$
\tilde{\varphi}_{c, J} \sigma_{d} \tilde{\varphi}_{c, J}^{+}=\sigma_{d-c}
$$

Since $\sigma_{d-c} \sigma_{d-c}=\sigma_{d-c}$ it follows immediately that one can say, with the same abuse of notation

$$
\tilde{\varphi}_{c, J} \sigma_{d} \tilde{\varphi}_{c, J}^{+} \sigma_{d-c}=\sigma_{d-c} .
$$

Optimality of compensation In the proposed control scheme, the input $u_{2}$ that is delivered to the plant is generated by the right inverse of the approximate stiction, this is $u_{2}=\tilde{\varphi}_{c, J}^{+} u_{1}$ although the plant has saturation and stiction at the input. Following the results of Theorem 1, the linear controller and the anti-windup can be designed based in the linear plant's dynamics $G$ and the saturation level $d-c$. This structure is optimal in the sense that it renders the best possible match between $u_{1}$ and $u_{4}$.

Theorem 2. Let $\tilde{\varphi}_{c, J}, \tilde{\varphi}_{c, J}^{+}$and $\sigma_{d}$ be defined as in the previous sections. Suppose $u_{1} \in L_{p}[0, \infty)$ with $p \geq 1$ and let

$$
u_{4}=\left(\tilde{\varphi}_{c, J} \sigma_{d} \tilde{\varphi}_{c, J}^{+}\right) u_{1} \text { with } u_{4}(0)=\operatorname{sat}_{d-c} u_{1}(0) .
$$

Then, for any causal $\Psi$ such that $\left(\tilde{\varphi}_{c, J} \sigma_{d} \tilde{\varphi}_{c, J}^{+}\right) u_{1} \in L_{p}[0, \infty)$

$$
\left\|u_{1}-\left(\tilde{\varphi}_{c, J} \sigma_{d} \Psi\right) u_{1}\right\|_{p} \geq\left\|u_{1}-u_{4}\right\|_{p} .
$$

\section{Proof. See [27].}

Theorem 2 shows that the structure proposed in Theorem 1 is optimal. Consider Fig. 11 with $u_{4}=\left(\tilde{\varphi}_{c, J} \sigma_{d} \tilde{\varphi}_{c, J}^{+}\right) u_{1}$. The relationship between $u_{1}$ and $u_{4}$ is referred to in Theorem 2 . Ideally, one would want to 'remove' the nonlinearities of the plant by making $u_{4}=u_{1}$ with some controller. However, when a saturation is present, additional to the stiction nonlinearity, this is not possible. In fact, the best possible approximation of $u_{4}$ to $u_{1}$ is $u_{4}=\sigma_{d-c} u_{1}$. Note that the resulting saturation is more restrictive than the original $\sigma_{d}$.

\section{Anti-windup example}

Theorem 1 implys that for a plant with saturation and a nonlinearity (in this case stiction) in the input, if the right inverse of the nonlinearity can be implemented, then the system can be seen as an input saturation problem that can be compensated using standard methods. In this section we show the applicability of our method using a very simple back-calculation anti-windup, noting that other, more complex configurations can be used.

The results are demonstrated in simulation using the previous example, which is taken from [10]. It is assumed that the plant is subject to saturation and stiction in the actuator. To compensate both nonlinealities an anti-windup controller is used in combination with the proposed inverse stiction.

The flow $P_{F}(s)$ and level $P_{L}(s)$ dynamics of the plant are repeated here for convenience.

$$
P_{F}(s)=\frac{1}{0.2 s+1} \quad P_{L}(s)=\frac{1}{15 s} e^{-s} .
$$

It is assumed that the plant (16) is subject to saturation and the approximate stiction in the actuator. The saturation $\sigma_{d}$ level is set to $d=2.5$, and the parameters of both the approximate stiction and its inverse are chosen as in the above example for the mild stiction case, this is $c=0.35$ and $J=0.3$.

The structure of the system and the controller is represented in Fig. 13. A simple anti-windup compensator is used for illustration purposes, however more specialized configurations can be used, [29, 30].

Two schemes are implemented in simulation as follows.

\section{Scheme 1:}

(a) PI (saturation active)

(b) PI with anti-windup (saturation active)

Scheme 1 does not include any stiction compensation.

\section{Scheme 2:}

(a) Saturation equivalence with PI with anti-windup (saturation active).

The gains of the PI in all cases are $K_{p}=5, T_{i}=5$ [10], with the gain in the anti-windup feedback set as $T_{a}=T_{i}$. The anti-windup augmentation compensates for the equivalent saturation $\sigma_{d-c}$, like in Fig. 13.

Scheme 1(a). The output of the input saturated system using a standard PI is shown in Fig. 14 (top). In this case, the output presents oscillations around the set point due to the presence of stiction nonlinearity and the saturation. The bottom part of the same figure shows the control signal, obtained from the PI, that is applied to the plant.

Scheme 1(b). Now, the saturated system subject to stiction nonlinearity is now controlled using a standard PI with anti-windup. The response and control signal of the 


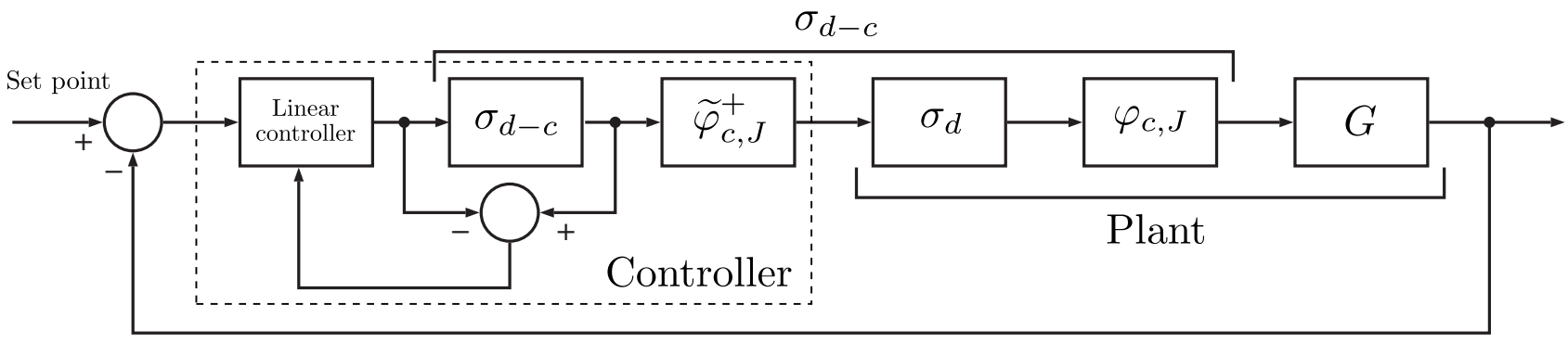

Figure 13. Proposed controller for a system subject to a stiction nonlinearity $\varphi_{c, J}$ and saturation $\sigma_{d}$ in the input using a linear controller with back-calculation anti-windup and approximate stiction right inverse. The anti-windup saturation $\sigma_{d-c}$ has a lower level than the plant saturation $\sigma_{d}$.
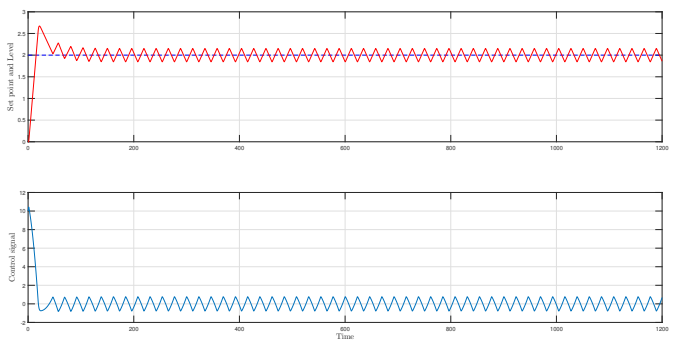

Figure 14. Scheme 1(a): System response of a plant with saturation and stiction in the input, using a standard PI controller (top) and the corresponding control signal, when no stiction compensation is used.
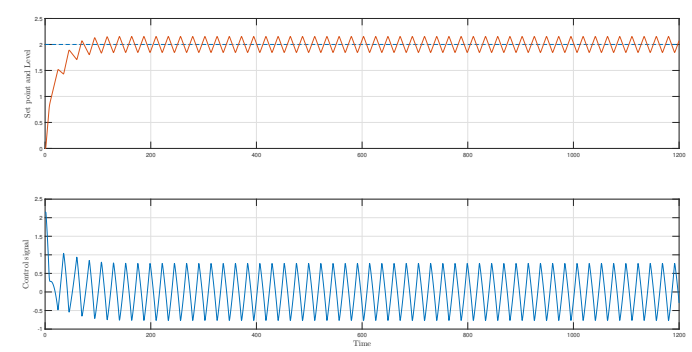

Figure 15. Scheme 1(b): System response of a plant with saturation and stiction in the input, using a standard PI controller with anti-windup (top) and the corresponding control signal, when no stiction compensation is used.

system is shown in Fig. 15, Compared to Fig. 14, the antiwindup compensator eliminates overshoot in the response and in the control signal, however it is not capable of removing oscillations in steady state, which are due to the stiction nonlinearity.

Scheme 2(a). In this case, the nonlinear dynamics of the plant are compensated using the proposed 'saturation equivalence' controller in combination with a PI with antiwindup. The closed-loop response is shown in Fig.16(top).


Figure 16. Scheme 2(a): System response of a plant with saturation and stiction in the input, using the 'saturation equivalence' controller with anti-windup (top) and the corresponding control signal.

It can be seen that the output correctly follows the reference in steady state.

\section{Demonstration with a standard stiction model}

To demonstrate the application of the saturation equivalence principle for stiction nonlinearity in a more general setting, simulations are performed using a standard stiction model reported by [9]. This model has been previously used to demonstrate compensation by approximate inversion of the stiction nonlinearity in [22].

In this section, it will be assumed that the plant described by the transfer functions (16) is subject to input saturation and stiction. Following [9, 22], the stiction nonlinearity is modeled as

$$
u_{4}=\left\{\begin{aligned}
u_{3}-f_{D} & \text { if } u_{3}-u_{4}^{-}>f_{S} \\
u_{3}+f_{D} & \text { if } u_{3}+u_{4}^{-}<-f_{S} \\
u_{4}^{-} & \text {if }\left|u_{3}-u_{4}^{-}\right| \leq f_{S}
\end{aligned}\right.
$$

where $u_{4}^{-}$represents the previous value of $u_{4}$. Parameters $f_{D}$ and $f_{S}$ represent the kinetic friction and the static friction bands [9], and are related to parameters $c$ and $J$, used by 


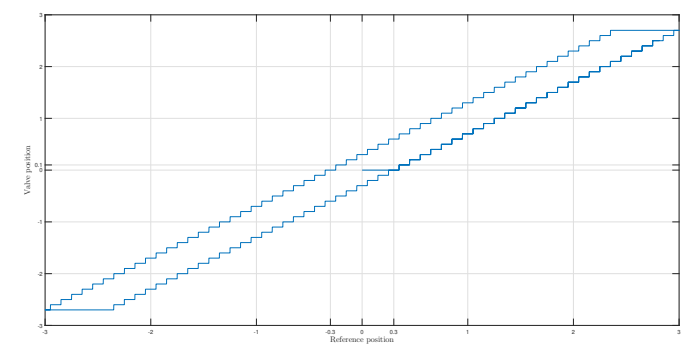

Figure 17. Reference versus valve position response for He's stiction model.

the stiction model proposed, by the following relations

$$
\begin{aligned}
& c=f_{D} \\
& J=f_{S}-f_{D}
\end{aligned}
$$

In this particular example, the stiction parameters are chosen as $f_{D}=0.25$ and $f_{S}=0.35$, which, according to equations (18)-(19), would correspond to $c=0.25$ and $J=$ 0.1 . However, as noted by [8], He's model is not an accurate representation of stiction during slipping motion since it presents a steplike behavior at this stage, thus causing the effective $f_{D}$ value to be larger than expected. This effect can be observed in Fig. 17 which shows the reference versus valve position response when using He's model with said parameters ( $c=0.25$ and $J=0.1$ ). From the figure, it can be observed that, due to inconsistencies in He's model, the acutal parameters that define stiction are $c=0.3$ and $J=0.1$, as opposed to what was orignally selected. The designer can, however, use the parameters observed in Fig. 17 to construct the inverse stiction, and achieve good results. This will be shown below. Note that in real life experiments, it will not be possible to measure the stiction parameters exactly, and therefore, an estimation will be needed. This issue is, however, outside of the scope of this paper. The reader is referred to [31], [5] for more information on advances in stiction quantification techniques.

Next, it is assumed that the saturation nonlinearity affecting the plant is characterized by $d=0.5$. Then, according to the saturation equivalence principle, the saturation nonlinearity $\sigma_{d-c}$ will be described by $d-c=$ $0.5-0.3=0.2$, as expected, see Fig. 18 , Notice, that the saturation principle holds even when the stiction model is not completely accurate. In this case, the approximate stiction inverse presented above is used to compensate the effects of the stiction nonlinearity. Note that the stiction inverse does not completely compensate for the steps present during sliding motion in He's model, which is expected, since the inverse is build for the case when jumps are only present when the input changes direction. To invert such motion would require an specific model to address such behavior. For example in [22] the authors propose

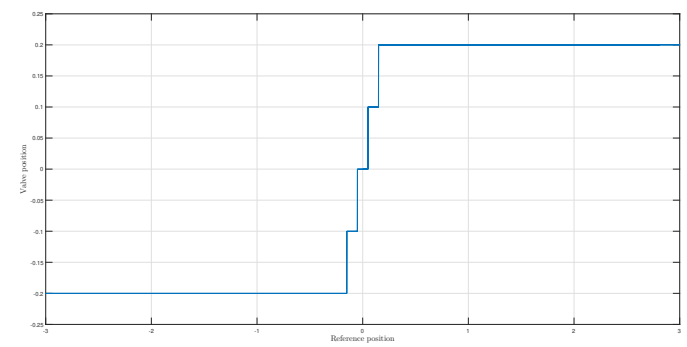

Figure 18. Reference versus valve position assuming saturation and stiction nonlinearity in the actuator. The approximate inverse stiction is used to compensate stiction.
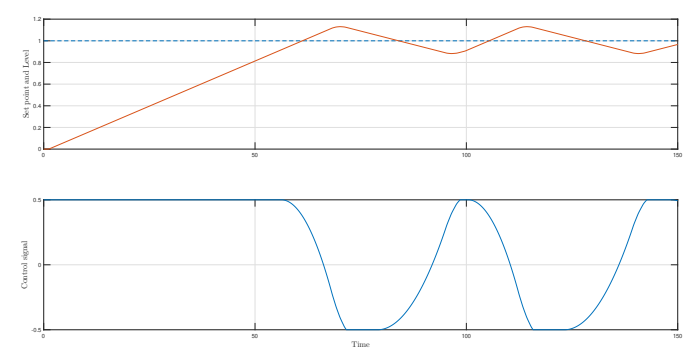

Figure 19. Case 1. System response of a plant with saturation and He's stiction, using a PI with antiwindup (top). Corresponding control signal, $u_{2}$ (bottom).

an approximate inverse for this particular stiction model. However, as will be shown next, the proposed 'saturation equivalence' method can still be used even when inversion is not perfect.

To illustrate the usage of the proposed scheme with an alternative stiction model in closed loop, a unit step reference is assumed for the plant (16), and the closed-loop model is build according to Fig.13, The linear controller is a PI with gains $K_{p}=3, T_{i}=5$, and the anti-windup feedback gain chosen as $T_{a}=T_{i}$. Two simulation cases are studied:

Case 1 PI with anti-windup (saturation active).

Case 2 Saturation equivalence with PI with anti-windup (saturation active).

Case 1. First the plant is supposed to be affected by saturation and stiction at the actuator. The nonlinear component of the plant is described by the stiction model proposed by $\mathrm{He}$ in [9] and reproduced in equation [17). Fig. 19 shows the closed-loop response when a unit step reference is applied to the system. Notice the wide oscillations around the set-point, generated by stiction.

Case 2. Next, the stiction nonlinearity is compensated by the approximate inverse stiction proposed in this work. Thus, the saturation equivalence principle is applied with a 

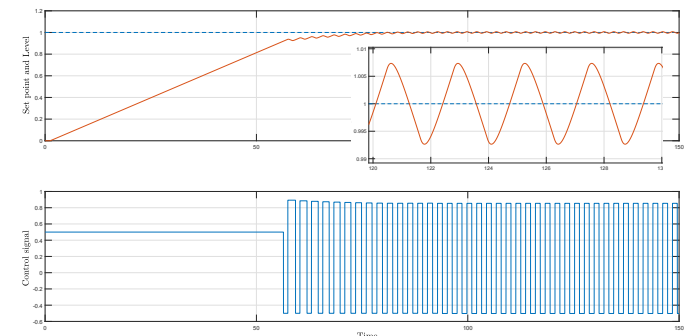

Figure 20. Case 2. System response for a plant with saturation and He's stiction using the saturation equivalence controller with the proposed approximate inverse stiction (top). Corresponding control signal, $u_{2}$ (bottom).

different stiction model, for which no inverse is explicitly designed. Fig. 20 shows the closed loop response in this case. The proposed approximate inverse does not completely cancel the effects of He's stiction. As explained previously, this is mainly due to the fact that He's model presents steplike behavior during slipping motion, and this type of behavior is not accounted for in the proposed inverse. However, notice that the saturation equivalence offers good performance in steady state. Fig. 20 also shows a close-up of the steady state response; it is clear from the figure that the error in this case is around \pm 0.007 . The control signal, on the other hand, presents chattering. This can be dealt with using some anti-chattering algorithm as suggested by [26] and [32].

\section{Demonstration with exact stiction}

In Section Anti-windup example, the plant was assumed to be subject to approximate stiction, and an appropriate inverse was build for such case. This section presents results for the case when the plant exhibits exact stiction but only an approximate stiction inverse can be used.

This example assumes that a sinusoidal reference $r(t)=$ $A \sin (\omega t)$, with $A=3$ and $\omega=0.005 \mathrm{rad} / \mathrm{s}$, must be followed by the plant described in (16). In this example the plant is affected by exact stiction and saturation. The stiction parameters are given as $c=0.25$ and $J=0.1$, while the saturation parameter is set as $d=0.5$.

As before, the results are organized in case form

Case 1. PI with anti-windup (saturation active).

Case 2. Saturation equivalence with PI with anti-windup (saturation active).

In both cases, the parameters for the PI are chosen as $K_{p}=3, T_{i}=5$, and the anti-windup feedback gain as $T_{a}=$ $T_{i}$.

Case 1. A PI controller with anti-windup is used to control the linear plant in (16) which is subject to saturation
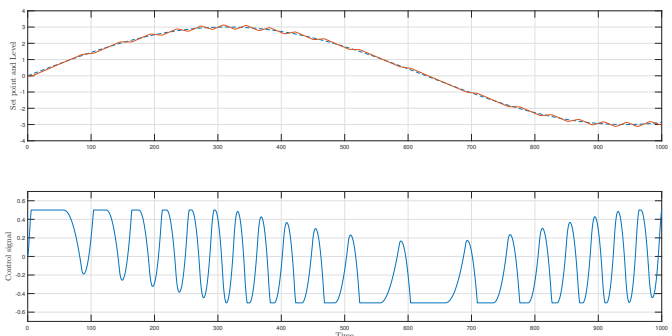

Figure 21. Case 1: The linear plant is subject to saturation and exact stiction. System response with sinusoidal reference, when only a PI with anti-windup is used (top). Corresponding control signal, $u_{2}$ (bottom).

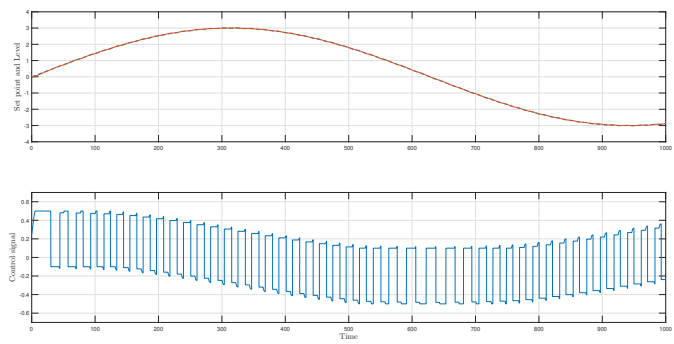

Figure 22. Case 2: The linear plant is subject to saturation and exact stiction. System response with sinusoidal reference when the saturation equivalence controller with anti-windup is used (top). Corresponding control signal (bottom).

and exact stiction. In this case, no compensation strategy for the stiction nonlinearity is considered. Fig. 21] shows the response, and the control signal $u_{2}$ for this case. Notice the persistent oscillations all along the response.

Case 2. Now, the saturation equivalence controller proposed here is implemented. An inverse of the approximate stiction is used with parameter $\varepsilon=1 \times 10^{-5}$. The inverse is combined with the same PI controller with anti-windup, and the results are shown in Fig.22, This time, the oscillations in the output signal are reduced, thanks to the presence of the inverse. Note, however, that although the amplitude of the oscillations is smaller than in Case 1, they are not completely eliminated. The reason for this is that exact stiction is a non invertible nonlinearity. Therefore, although parameter $\varepsilon$ can be varied to adjust precision, when the exact stiction, with vertical jumps is considered, the effect of $\varepsilon$ is minimal. Its effect is better appreciated when paired with an almost exact stiction, as would in fact happen in the real world. In that case, $\varepsilon$ can be adjusted (tipically, reduced) to obtain a better approach. Notice as well that the control signal is affected by chattering, then, it is suggested that an anti-chattering solution is used, as in [26] and [32]. 


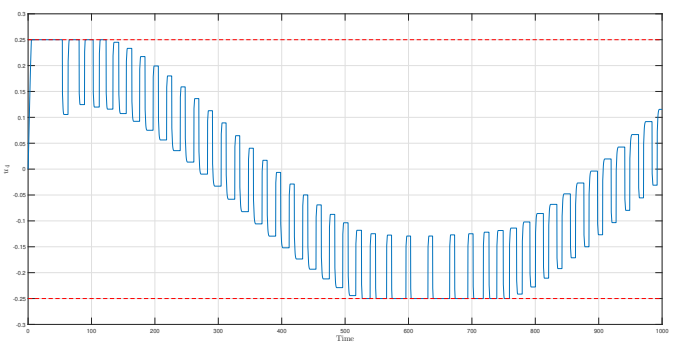

Figure 23. The signal $u_{4}$ complies with the saturation equivalence principle in spite of the presence of non invertible exact stiction.

Nevertheless, although exact stiction cannot be inverted, the saturation equivalence principle prevails. Recall Fig.[13, where signals $u_{1}$ to $u_{4}$ are defined, and note that according to the saturation equivalence principle, one would expect signal $u_{4}$ to be bounded by a saturation $\sigma_{d-c}$ with $d-c=$ 0.25 . This fact can be appreciated in Fig. 23.

\section{Conclusions}

A compensation scheme is proposed for plants with saturation and stiction characteristics at the input. The model for stiction in based on data-driven models. An approximation for stiction is introduced, considering the fact that the exact stiction will not happen in real life systems. Then a right inverse of the approximate stiction nonlinearity to compensate for stiction is proposed. The approximation of stiction is done through the design parameter $\varepsilon$, then it is possible to adjust the right inverse to effectively cancel the nonlinearity. Note that it is in fact reasonable to use this approximation since, real life systems, will not exhibit exact stiction, namely, with jumps of vertical slope. As such, parameter $\varepsilon$ is useful when measurements of the slope are not readily available (the typical case).

The 'saturation equivalence' principle has been previously formalized for backlash nonlinearity by the authors in [27]. Then, the main contribution of this article is to show that this principle holds for stiction as well, despite this nonlinearity not having an exact inverse. The 'saturation equivalence' method can be used for plants with saturation and stiction in the input, by building an approximation of the inverse stiction. It has been shown, in simulation, that the proposed inverse can be used with different stiction models, and that the 'saturation equivalence' principle holds when considering a non invertible, exact stiction model.

The power of the 'saturation equivalence' method is that it opens the door for simple controllers for input saturated system, such as PI (PID) with anti-windup or basic MPC [24], to be used to compensate for complex dynamic nonlinearities, in this case stiction.

This work includes two case studies where the proposed method is demonstrated in the situation where the inverse nonlinearity does not coincide exactly with the stiction present in the plant. This is shown by (a) using a standard stiction model, e.g. [9], and (b) using an exact stiction, this is, when the jumps in the nonlinearity have vertical slope. In the first case, a unit step reference is assumed, and in the second, the objective is to track a sine wave reference. In both examples, tracking is achieved, and the oscillations are significantly reduced despite the non availability of an exact inverse. This, ultimately, prevents the approximate inverse from eliminating the oscillations completely. However, as evidenced by the results, the 'saturation equivalence' principle holds for both cases, and therefore, a classical controller for input saturated systems, such as PI with antiwindup can be used, in general.

Although we have adopted a data driven model, we have not addressed the problem of acquiring the parameters of the nonlinearity. Detection and identification is an important practical problem, but beyond the scope of this work.

\section{Acknowledgements}

Authors would like to thank CONACyT for the funding provided for the realization of this work. Authors also thank the reviewers for their valuable comments and thorough revision, which have helped to improve the quality of this paper.

\section{References}

[1] Fisher Controls. Control valve handbook. Fourth ed. Fisher Controls International LCC, 2005.

[2] Jelali M and Huang B. Detection and Diagnosis of Stiction in Control Loops: State of the Art and Advanced Methods. Advances in Industrial Control, Springer London, 2009.

[3] Choudhury A, Thornhill N and Shah S. Modelling valve stiction. Control Engineering Practice 2005; 13(5): 641-658.

[4] Hägglund T. A friction compensator for pneumatic control valves. J Process Control 2002; 12: 897-904.

[5] di Capaci RB and Scali C. Review and comparison of techniques of analysis of valve stiction: From modeling to smart diagnosis. Chemical Engineering Research and Design 2018; 130: 230 - 265.

[6] Ivan L and Lakshminarayanan S. A new unified approach to valve stiction quantification and compensation. Ind Eng Chem Res 2009; (7): 34743483. 
[7] Srinivasan R and Rengaswamy R. Approaches for efficient stiction compensation in process control valves. Comput Chem Eng 2008; 32: 218-229.

[8] Chen S, Tan K and Huang S. Two-layer binary tree data-driven model for valve stiction. Ind Eng Chem Res 2008; 47: 2842-2848.

[9] He Q, Wang J, Pottmann $M$ et al. A curve fitting method for detecting valve stiction in oscillating control loops. Ind Eng Chem Res 2007; 46: 45494560 .

[10] Kano M, Maruta H, Kugemoto $\mathrm{H}$ et al. Practical model and detection algorithm for valve stiction. pp. 859 - 864. 7th IFAC Symposium on Dynamics and Control of Process Systems 2004 (DYCOPS -7), Cambridge, USA, 5-7 July, 2004.

[11] Xie L, Cong Y and Horch A. An improved valve stiction simulation model based on isa standard tests. Control Engineering Practice 2013; 21(10): 1359 1368.

[12] Wang J and Zhang Q. Detection of asymmetric control valve stiction from oscillatory data using an extended hammerstein system identification method. Journal of Process Control 2014; 24(1): 1 - 12.

[13] Armstrong-Hèlouvry B, Dupont P and de Wit C. A survey of models, analysis tools and compensation methods for the control of machines with friction. Automatica 1994; 30(7): 1083-1138.

[14] Farenzena M and Trierweiler J. Modified PI controller for stiction compensation. IFAC Proceedings Volumes 2010; 43(5): 799-804. 9th IFAC Symposium on Dynamics and Control of Process Systems.

[15] de Souza L Cuadros A, Munaro C and Munareto S. Improved stiction compensation in pneumatic control valves. Computers $\mathcal{E}$ Chemical Engineering 2012; 38(5): 106-114.

[16] Wang T, Xie L, Tan F et al. A new implementation of open-loop two-move compensation method for oscillations caused by control valve stiction. IFACPapersOnLine 2015; 48(8): 433 - 438. 9th IFAC Symposium on Advanced Control of Chemical Processes ADCHEM 2015.

[17] Tang L and Wang J. Estimation of the most critical parameter for the two-movement method to compensate for oscillations caused by control valve stiction. IEEE Transactions on Control Systems Technology 2016; 24(5): 1869-1874.
[18] Mohammad MA and Huang B. Compensation of control valve stiction through controller tuning. Journal of Process Control 2012; 22: 1800-1819.

[19] Fang L, Wang J, Tan X et al. Analysis and compensation of control valve stiction-induced limit cycles. In 2016 IEEE International Conference on Advanced Intelligent Mechatronics (AIM). pp. 16881693.

[20] Zabiri H and Samyudia Y. MIQP-Based MPC in the presence of control valve stiction. Chemical Product and Process Modeling 2009; 4(3). Article 7.

[21] Durand H and Christofides PD. Actuator stiction compensation via Model Predictive Control for nonlinear processes. AIChE Journal 2016; 62(6): 2004-2016.

[22] di Capaci RB, Vaccari M and Pannocchia G. A valve stiction tolerant formulation of $\mathrm{mpc}$ for industrial processes. IFAC-PapersOnLine 2017; 50(1): 9044 9049. 20th IFAC World Congress.

[23] Novak J and Chalupa P. Compensation of valve deadzone using mixed integer predictive control. In 2017 31st European Conference on Modelling and Simulation. Budapest, Hungary, pp. 1-5.

[24] Rodríguez-Liñán MC and Heath WP. MPC for plants subject to saturation and deadzone, backlash or stiction. IFAC Proceedings Volumes 2012; 45(17): 418 - 423. 4th IFAC Conference on Nonlinear Model Predictive Control.

[25] Rodríguez-Liñán MC and Heath WP. Controller structure for plants with combined saturation and deadzone/backlash. In 2012 IEEE International Conference on Control Applications. pp. 1394-1399.

[26] Rodríguez-Liñán MC. New control design and analysis techniques for plants with actuator nonlinearities. Phd thesis, School of Electrical and Electronic Engineering, University of Manchester, 2013.

[27] Rodríguez-Liñán MdC and Heath WP. Backlash compensation for plants with saturating actuators. Proceedings of the Institution of Mechanical Engineers, Part I: Journal of Systems and Control Engineering 2017; 231(6): 471-480.

[28] Tao G and Kokotović P. Adaptive control of systems with actuator and sensor nonlinearities. John Wiley and Sons, 1996.

[29] Tarbouriech S and Turner M. Anti-windup design: an overview of some recent advances and open problems. IET Control Theory A 2007; 3(1): 1-19. 
[30] Zaccarian L and Teel AR. Modern Anti-windup Synthesis. Princeton, 2011.

[31] Choudhury A, Shah S and Thornhill N. Diagnosis of Process Nonlinearities and Valve Stiction. 1 ed. Advances in Industrial Control, Springer-Verlag Berlin Heidelberg, 2008.

[32] Johansson KH, Egerstedt M, Lygeros J et al. On the regularization of zeno hybrid automata. Systems $\mathcal{F}$ Control Letters 1999; 38(3): 141-150. 